\title{
Lattice Mismatch Drives Spatial Modulation of Corannulene Tilt on $\mathrm{Ag}(111)$
}

Anu Baby, ${ }^{\dagger}$ He Lin $,{ }^{\dagger}, \#$ Abhilash Ravikumar, ${ }^{\dagger}$ Carla Bittencourt, ${ }^{\ddagger}$ Hermann A. Wegner, "Luca Floreano, ${ }^{*, \S}$ Andrea Goldoni, ${ }^{*} \|$ and Guido Fratesi*, ${ }^{*}$

$\dagger$ Università degli Studi di Milano-Bicocca, Dipartimento di Scienza dei Materiali, via Cozzi 55, I-20125, Milano, Italy

$\ddagger$ Chimie des Interactions Plasma-Surface, University of Mons, 20 Place du Parc, 7000 Mons, Belgium

9Institute of Organic Chemistry, Justus Liebig University Giessen, Heinrich-Buff-Ring 17, 35392 Giessen, Germany

$\S C N R-I O M$, Laboratorio TASC, Basovizza SS-14, Km 163.5, I-34149 Trieste, Italy

|| Elettra Sincrotrone Trieste, s.s. 14 km 163.5 in Area Science Park, 34149 Trieste, Italy and INSTM - Elettra, Lab. Micro 83 Nano-Carbon, s.s. 14 km 163.5 in Area Science Park, 34169 Trieste, Italy

$\perp$ Dipartimento di Fisica, Università degli Studi di Milano, via Celoria 16, I-20133, Milano, Italy

\#Current address: Institute of Applied Chemistry, Xinjiang University, Urumqi, 830046 Xinjiang, P. R. China

E-mail: floreano@iom.cnr.it; andrea.goldoni@elettra.eu; guido.fratesi@unimi.it 


\begin{abstract}
We investigated the adsorption of corannulene $\left(\mathrm{C}_{20} \mathrm{H}_{10}\right)$ on the $\mathrm{Ag}(111)$ surface by experimental and simulated scanning tunneling microscopy (STM), X-ray photoemission (XPS) and near-edge X-ray absorption fine structure (NEXAFS). Structural optimizations of the adsorbed molecules were performed by density functional theory (DFT) and the core excited spectra evaluated within the transition potential approach. Corannulene is physisorbed in a bowl-up orientation displaying a very high mobility (diffusing) and dynamics (tilting and spinning) at room temperature. At the monolayer saturation coverage, molecules order into a close compact phase with an average intermolecular spacing of $\sim 10.5 \pm 0.3 \AA$. The lattice mismatch drives a long wavelength structural modulation of the molecular rows, which, however, could not be identified with a specific superlattice periodicity. DFT calculations indicate that the structural and spectroscopic properties are intermediate between those predicted for the limiting cases of an on-hexagon geometry (with a three-fold, $\sim 8.6 \AA$ unit mesh) and an onpentagon (with a four-fold, $\sim 11.5 \AA$ unit mesh). We suggest that molecules smoothly change their equilibrium configuration along the observed long wavelength modulation of the molecular rows by varying their tilt and azimuth in between the geometric constraints calculated for molecules in the three-fold and four-fold phases.
\end{abstract}

\title{
Introduction
}

The study of curved poly- and heteroaromatic molecules is relevant to supramolecular chemistry, due to the possibility of heteromolecular convex-to-concave coupling, ${ }^{1-3}$ which may improve the $\pi-\pi$ coupling, hence the intermolecular electron transport, as reported for a few shape-matching heteromolecular systems. ${ }^{4,5}$ The interaction of open geodesic polyarenes with substrates may further enhance and stabilize the convex-to-concave heteromolecular coupling. ${ }^{2}$ In addition, buckybowl aromatic molecules have been shown to transfer chiral enantioselectivity to an otherwise achiral surface. ${ }^{6}$ In this regard, corannulene, the simplest 
bucky-bowl fullerene fragment, displays very intriguing behavior (e.g. up/down bowl flipping, ${ }^{7}$ polymorphism ${ }^{8}$ and enantiomorphism ${ }^{9}$ by bowl tilting) when deposited on crystalline surfaces due to its $\mathrm{C}_{5 v}$ symmetry that cannot match any crystal symmetry.

The bowl-shaped structure of corannulene, $\mathrm{C}_{20} \mathrm{H}_{10}$, can be understood as a 20-atom section of $\mathrm{C}_{60}$ fullerene, comprised of a central pentagonal ring surrounded by five hexagonal rings. ${ }^{10,11}$ From the electronic point of view, the corannulene C-C bond lengths suggest significant $\pi$-delocalization in the system. For instance, acceptance of four electrons ${ }^{12}$ makes corannulene more electron-rich per carbon atom than the commonly used electron acceptor $\mathrm{C}_{60} \cdot{ }^{13}$ Recently, a practical synthetic route made corannulene available for comprehensive studies, which has also boosted its utilization in a wide range of applications. ${ }^{14}$ Indeed, corannulene has been independently reported to be an excellent material for electron conductivity applications ${ }^{15-19}$ and its electron-accepting properties can be tuned through functionalization of the $\mathrm{C}_{20} \mathrm{H}_{10}$ bowl, possibly favoring a bowl-in-bowl coupling which facilitates intermolecular electron hopping. ${ }^{20}$

To answer some of the questions concerning $\pi-\pi$ stacking interactions between curved systems, many ab initio calculations have been performed on corannulene dimer motifs with a major emphasis on the relative importance of $\pi-\pi$ interactions between bowl-shaped systems in comparison with those in planar aromatic systems. For the curved corannulene dimers, eclipsed conformations are preferred while a staggered coupling is preferred for planar dimers. ${ }^{21-24}$

From an experimental point of view, a few scanning tunneling microscopy (STM) and photoemission experiments of this molecule have been performed on the $\mathrm{Cu}(111)$ and $\mathrm{Cu}(110)$ surfaces. $^{2,8,9}$ A general trend can be rationalized, where the molecules adsorb in a concave upwards configuration in order to maximize the overlap between the $\pi^{*}$ system of one of the aromatic rings with the charge density of the metal substrate. The symmetry of the substrate ultimately dictates the specific adsorption geometry, similar to the case of $\mathrm{C}_{60} \cdot{ }^{25}$ On the rectangular (110) unit cell, 2D ordered domains are formed by adsorption on a carbon- 
carbon bond at the rim of the central pentagon, yielding a slightly tilted geometry. ${ }^{2,9}$ On the $\mathrm{Cu}(111)$ close compact surface, corannulene forms a hexagonal 2D lattice by facing the substrate with a hexagon (like $\mathrm{C}_{60}{ }^{26}$ ), again in a tilted geometry. ${ }^{8}$ Direct adsorption on the pentagonal ring has only been observed for isolated molecules or in disordered areas. ${ }^{8}$ In fact, the mismatch between the $C_{5 v}$ molecular symmetry and any crystalline substrate is relieved by molecular tilting in $2 \mathrm{D}$ ordered phases. ${ }^{27}$ However, very recent STM investigations of corannulene on $\mathrm{Ag}(111)$ rather claimed the coexistence of randomly distributed bowl-up and bowl-down oriented molecules at low temperature $(77 \mathrm{~K})$ in an otherwise ordered molecular array displaying an oblique symmetry with an occupancy of one molecule every four substrate lattice units. ${ }^{7}$ There, the occurrence of bowl-down molecules has been tentatively attributed to a strong coupling to silver that would prevent the flipping of molecules that landed in a concave downwards configuration. Most importantly, such a morphology would yield paramount consequences for the growth of further molecular layers, as the coupling with second layer is strictly determined by the orientation of the first layer molecules. ${ }^{28-35}$

Here we report a combined structural and electronic investigation of the corannulene adsorption on $\operatorname{Ag}(111)$ by STM, X-ray photoemission (XPS) and near-edge X-ray absorption spectroscopy (NEXAFS). Experimental evidences are interpreted by simulations performed with density functional theory (DFT) calculations. Molecules order into an incommensurate close-compact phase with an intermolecular spacing of $\sim 10.5 \AA$ that is intermediate between the periodicity of a three-fold and a four-fold phase. Since the experimental evidences point to the coexistence of molecules with different configurations (tilt and azimuth), we modelled corannulene in the commensurate phases corresponding to the two limiting periodicities. DFT calculations predict molecules to adsorb on a hexagon face (tilted geometry) for the compressed $(3 \times 3)$ phase, while corannulene prefers standing on the pentagon face (bowlup geometry) for the widely spaced $(4 \times 4)$ phase. A deeper understanding of the tilting geometry can be obtained by NEXAFS measurements with polarized light. The theoretical analysis points out a non-trivial dependence of polarized NEXAFS intensities on adsorption 
angle due to the molecular curvature; also keeping this into account, the measured effective tilt angle indicates the occurrence of a contribution from a fraction of tilted molecules. Both the structural and electronic properties of the monolayer phase are found to be intermediate between those of the molecules in the two limiting phases.

\section{Experimental and theoretical methods}

\section{Experimental details}

The measurements have been carried out in ultra-high-vacuum (base pressure $3 \times 10^{-10} \mathrm{mbar}$ ) at the IOM-Elettra joint laboratory for microscopy OSMOS and at the ALOISA beamline of the Elettra synchrotron light source (Trieste, Italy). C 1s XPS was measured at $h \nu=500 \mathrm{eV}$ with an overall energy resolution of $240 \mathrm{meV}$. The binding energy was calibrated to the $\mathrm{Ag}$ $3 \mathrm{~d}_{5 / 2}$ peak at $368.3 \mathrm{eV}$. NEXAFS was taken at partial electron yield in an energy range of 280-320 eV with a resolution of $80 \mathrm{meV}$. We measured spectra in transverse magnetic (TM), and transverse electric (TE), polarization (corresponding to quasi-p- and s-polarization, respectively) by rotating the surface around the photon beam axis, while keeping the incidence angle at a constant grazing value of $6^{\circ}$. Further details about scattering geometry, photon energy calibration and spectra normalization can be found in Ref. ${ }^{36}$

STM measurements have been performed with an Aarhus type microscope that is connected to a preparation chamber equipped with a LEED apparatus. We collected topographic images at room temperature with a tungsten tip after deposition of increasing coverage up to the full saturation of the monolayer. Molecules were only imaged at a positive sample bias, while negative bias systematically yielded fuzzy images also at full monolayer coverage, possibly due to strong coupling of the tip to the molecules. The length scale has been calibrated on atomically resolved images of both the clean $\mathrm{Ag}(111)$ surface (Figure S1 in the Supporting Information) and a reference $\mathrm{Au}(111)$ surface, as measured in a range of scale length from 5 to $15 \mathrm{~nm}$. 
The $\operatorname{Ag}(111)$ surface was cleaned by repeated $\mathrm{Ar}^{+}$sputtering at $1.5 \mathrm{keV}$ at room temperature for 30 minutes, followed by thermal annealing up to $800 \mathrm{~K}$. Corannulene molecules were evaporated from a homemade boron nitride crucible at about $400-410 \mathrm{~K}$. In both experimental setups, the rate has been calibrated by means of a quartz microbalance and verified either by XPS or by STM. At room temperature, corannulene forms a saturation layer on the $\operatorname{Ag}(111)$ surface at an equivalent thickness of $\sim 2.5 \AA$, which we define as 1 monolayer (ML) coverage. At the low deposition rates considered here, 0.1-0.2 $\AA / \mathrm{min}$, we have not observed the condensation of next layer molecules. This points either to extremely high mobility of molecules, hence forming widely spaced 3D clusters which escape detection by surface sensitive XPS technique, or to a very small sticking coefficient due to the mismatch between the orientation of the first molecular layer and that of the natural growth direction of the molecular crystal planes. Such a behavior has been reported also for other polyconjugated hydrocarbons of similar size, e.g. perylene on $\mathrm{TiO}_{2}(110) \cdot{ }^{37}$

\section{Computational details}

In this work, we have used the open source codes from the QUANTUM ESPRESSO distribution. ${ }^{38,39}$ Calculations were based on DFT using the non-local optB88-vdW exchange functional. ${ }^{40}$ Ultra-soft pseudopotentials and plane-wave basis sets were used with a planewave cutoff of 27 Ry. The supercell was constructed with a Ag slab having three layers with the molecule adsorbed only on one side, and was periodically repeated in all three directions. A vacuum space of $22 \AA$ was used between the replicated slabs along the surface normal. Hexagonal surface unit cells of size $3 \times 3$ and $4 \times 4$ were used to model high- and low-coverage phases, respectively. A $12 \times 12$-equivalent Monkhorst Pack special k-points mesh ${ }^{41}$ was used.

The dependence of C 1s XPS on the initial state was evaluated by determining the core level shifts (CLS) between the inequivalent carbon atoms. Following Ref., ${ }^{42}$ the CLS was determined by the total energy differences between the calculations with a $\mathrm{C}$ pseudopotential generated with a full core hole (FCH) at the 1s level for the different inequivalent atoms and 
their weighted average. For determining NEXAFS, we have taken the transition-potential (TP-DFT) approach introduced by Triguero et al. ${ }^{43,44}$ In this method, one performs a selfconsistent calculation of the effective Kohn-Sham potential with a half-core-hole (HCH) occupation of the initial-state 1 s orbital and typically neglects the fractional occupation of the final state (but the total charge of the system is set to neutral here due to the screening from metal). The $\mathrm{HCH}$ pseudopotential used here required a higher plane wave cutoff of 59 Ry. The explicit calculation of the unoccupied orbitals to evaluate the spectrum was avoided following a Lanczos technique as implemented in the XSPECTRA code ${ }^{45}$ of QUANTUM ESPRESSO. This setup allows us to estimate efficiently the spectrum also in the continuum energy region, where the detail of transitions to $\sigma^{*}$ states can be used to determine the azimuthal orientation of the molecules ${ }^{37,46}$ and has been validated by the comparison to experiments in a variety of cases. ${ }^{37,47-49}$ We finally remark that by using a pseudopotential approach, as the absolute energies are inaccessible, the XPS and NEXAFS energy scales are offset from the true energy scales by an undetermined but fixed constant. STM images have been simulated by the Tersoff-Hamann method ${ }^{50}$ evaluating the local density of states corresponding to the energy window from the Fermi energy to an applied bias $V=+1 \mathrm{~V}$.

\section{Results and discussion}

\section{STM topography and electron diffraction}

We observed a very high molecular mobility at room temperature, which prevented the imaging of individual molecules on terraces in the submonolayer range. Up to at least 0.4 ML coverage, we observe fuzzy stripes parallel and adjacent to steps and fuzzy domains surrounding point defects. We attribute this to molecular condensation (decoration) at both point and line defects. However, molecules display a very large mobility, as witnessed by the change of stripe definition as a function of the relative scanning speed (detailed images are shown in Figure $\mathrm{S} 2$ of the Supporting Information). In addition, fast diffusing isolated 
molecules systematically prevented the acquisition of atomic resolution on the uncovered $\operatorname{Ag}(111)$ regions. To take advantage of the preferential interaction with steps (both above and below the edge), we also deposited molecules on surfaces pre-patterned with vacancy islands in order to slow down the effective mobility for a better imaging of the molecular contrast and orientation. Vacancy islands with monoatomic step on terraces were produced by low energy $\mathrm{Ar}^{+}$bombardment $(250 \mathrm{~V})$ with low fluence $(<1 \mu \mathrm{A})$ at room temperature (or slightly higher). This technique can be regarded as an inverse growth process that obeys the conventional mechanism of nucleation and growth and is effective on most substrates. ${ }^{51}$ For fcc(111) surfaces, the vacancy islands are mostly hexagonal or triangular. ${ }^{52}$ Molecules within small vacancy islands display a lower mobility and we could image the step edge restructuring due to the interaction with diffusing molecules, as shown in Figure S3 of the Supporting Information file and corresponding STM movie. In one specific case, molecules were trapped in a perfectly static triangular domain, as shown in Figure 1. While the mobility of molecules within the triangular vacancy island appears to be quenched, the high mobility of the molecules diffusing on the terrace is clearly appreciated from the appearance of the molecules condensed at the upper edge of the vacancy island. The fading intensity of the molecular rows away from the island edges can be associated with the corresponding decrease of the residence time at the domain border. Along with the same issue, one can also appreciate molecular condensation being smaller at the triangle apex than at the middle of the triangle edges, indicating that intermolecular attraction is dominant over molecule to substrate interaction. A similar transient morphology of molecular aggregation was originally observed for $\mathrm{CO}$ island nucleation at point defects, ${ }^{53}$ and a very similar behavior has been reported at low coverage also for coronene (the flat counterpart of corannulene, with a hexagon at the molecular center) on the same $\operatorname{Ag}(111)$ surface. ${ }^{54}$ We remark that molecules both within and outside the triangular vacancy island display the same regular periodicity of $\sim 10.5 \AA$, suggesting that the edge length of the vacancy island is closely commensurate to the intermolecular spacing. In addition, the molecules display an asymmetric contrast 
that might be associated with a tilted rim of the corranulene bowl. Molecules are observed to condense also at other defects, such as the protrusion visible in the bottom left corner of Figure 1. In this case, the molecules are less localized and the intramolecular asymmetric contrast can be identified only on a few molecules, which however do not display a coherent tilting, like those trapped at the vacancy island.

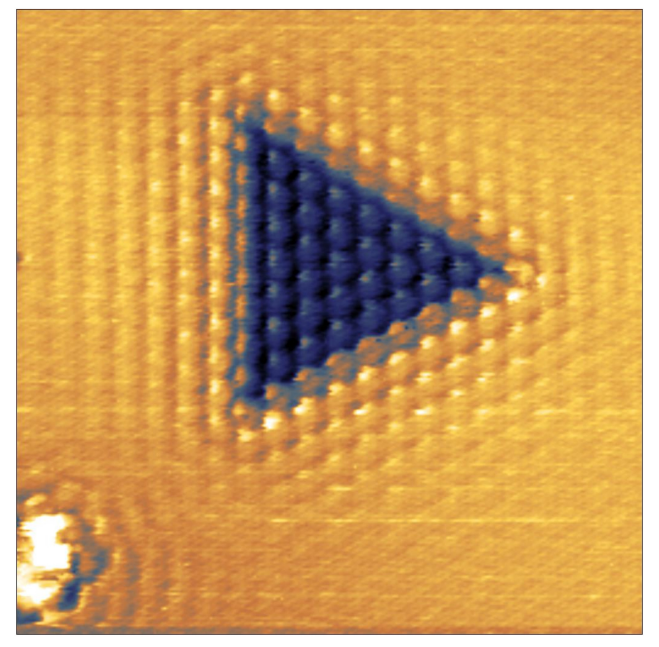

Figure 1: Experimental STM showing corannulene molecules adsorbed at a vacancy island. Image taken at a coverage of $0.3-0.4 \mathrm{ML}$ with sample bias $=+1.4 \mathrm{~V}, I=160 \mathrm{pA}$, image size $20 \times 20 \mathrm{~nm}^{2}$; molecules inside the triangular vacancy islands are trapped in a static configuration displaying an intermolecular spacing of $\sim 10.5 \AA$.

At the completion of the monolayer, molecules are arranged in close-compact arrays of equivalent entities, which are aligned along the high symmetry directions without displaying significant intramolecular features despite the good resolution and quality of the STM images, as judged by the sharpness of the molecular edges that can be seen in Figure 2. We were able to observe occasionally a weak central depression (either due to the relatively faster scanning speed or to a temporary change of the tip state), as may be detected in the small scale image of Figure 2(a), thus suggesting a bowl-up molecular orientation according to calculations for free molecules ${ }^{9}$ and to low-temperature STM measurements. ${ }^{7}$ We remark that this applies consistently to all the molecules, which would exclude the coexistence of corannulene molecules with opposite concavity. The molecules are regularly spaced with an average separation of $\sim 10.5 \AA$ (with some scattering of $\pm 0.3 \AA$ depending on the image 
scale), that corresponds to $\approx 3.6 \times$ the $\mathrm{Ag}(111)$ lattice constant of $2.88 \AA$.
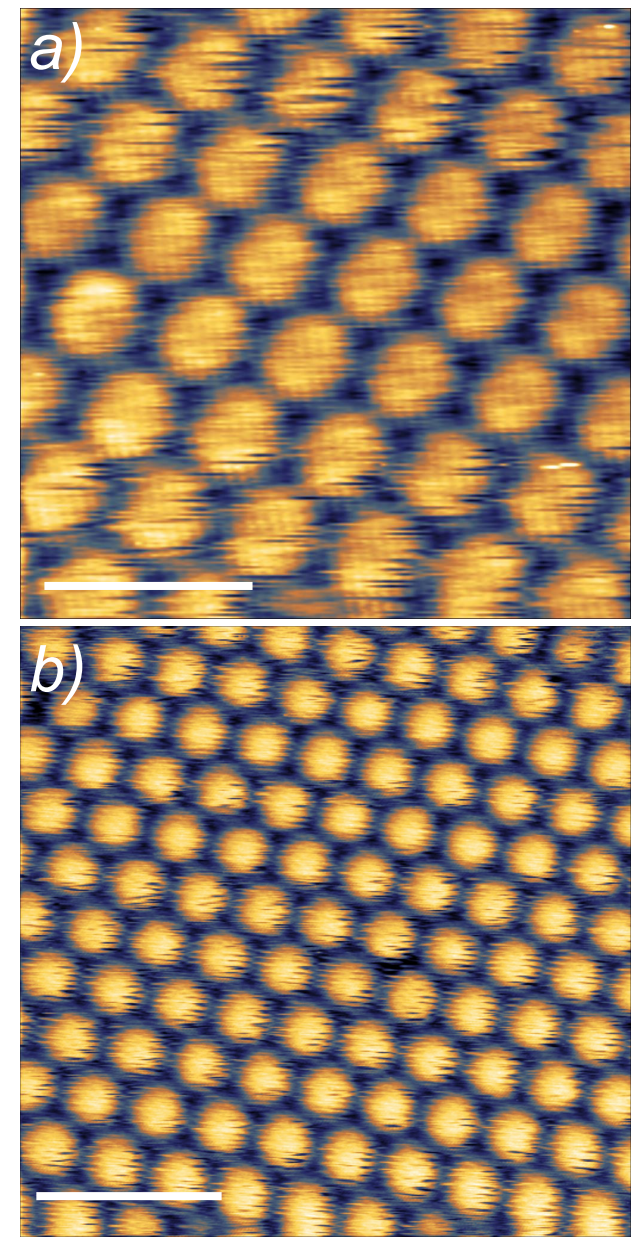

Figure 2: STM images at the monolayer saturation coverage displaying the formation of close-compact, although incommensurate, domains oriented along the substrate high symmetry directions with an average intermolecular spacing of $\sim 10.5 \AA$. A weak central depression can be appreciated in the molecules acquired at relatively faster scanning speed, as reported in the image (panel a: sample bias $=+2 \mathrm{~V}, I=440 \mathrm{pA}$, scan speed $0.9 \mathrm{~nm}^{2} / \mathrm{s}$, scale bar $=2 \mathrm{~nm}$ ). Otherwise, all molecules are equivalent, displaying a close-compact ordering (panel b: $+1.6 \mathrm{~V}, 640 \mathrm{pA}$, scan speed $0.4 \mathrm{~nm}^{2} / \mathrm{s}$, scale bar $\left.=3 \mathrm{~nm}\right)$.

Most remarkably, in large scale images measured at fast scanning speed, we sometimes observed the presence of a long wavelength weak intensity modulation within the molecular rows, as shown in Figure 3(a). We attribute such a modulation to the mismatch between the substrate lattice and the intermolecular spacing. The variation of molecular appearance, which is typically smeared out at slow scanning speed, can be better appreciated by comparing brighter and darker segments of adjacent rows. We observed an overall larger 
amplitude of the vertical contrast in the brighter segment of the molecular rows with respect to the darker ones, as highlighted in the vertical profile shown in the bottom panel of Figure 3(b). Overall the variation of the molecular contrast is very similar to that observed on $\mathrm{Cu}(111)$, where the correlated wiggling of molecules was shown to drive a two-dimensional phase transition. ${ }^{8}$
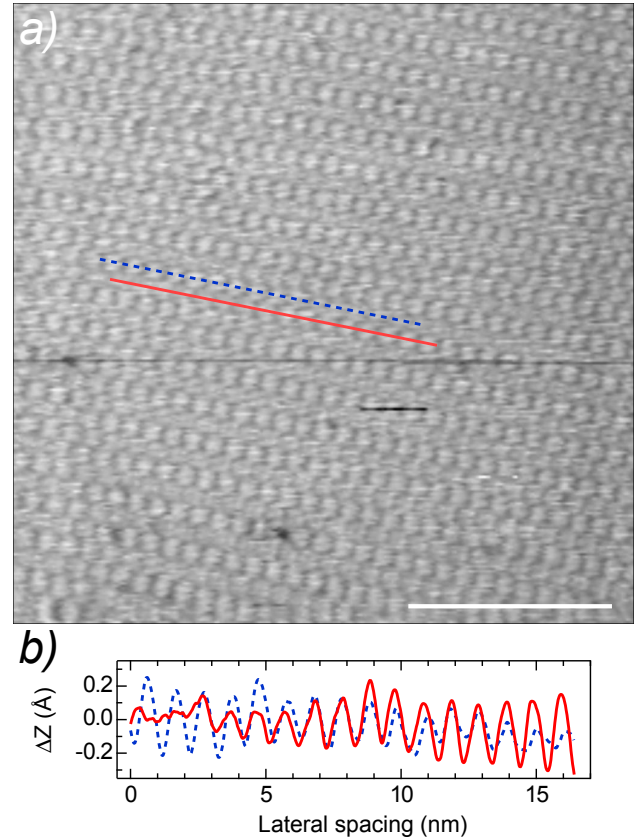

Figure 3: (a) STM image of the the same surface as in Figure 2 measured at a larger length scale $\left(+2.15 \mathrm{~V}, 80 \mathrm{pA}\right.$, scanning speed $\sim 5.5 \mathrm{~nm}^{2} / \mathrm{s}$, scale bar $\left.=10 \mathrm{~nm}\right)$ and displaying a long wavelength modulation of the contrast along the molecular rows. (b) Vertical profile along two adjacent molecular rows (see panel a) with opposite contrast: both molecular rows switch the molecular appearance in the middle region.

Both the vanishing of the long wavelength modulation and the weakness/absence of intramolecular contrast upon decreasing the scanning speed (hence increasing the tip residence time) point to a fast dynamical motion around the equilibrium position involving spatial displacement and tilt/azimuth rotation, and to a shallow interaction with the substrate. Dynamical flipping of the concavity at room temperature seems unlikely, as well as the coexistence of corrannulene molecules with opposite concavity, see Figure 2(a). The long wavelength modulation might be rather associated with a smooth modulation of the preferential molecular tilt and azimuth along the rows, which would be smeared by tip in- 
teraction in images measured at low scanning speed. In any case, we have not been able to detect either a specific periodicity of this intramolecular contrast modulation along the molecular rows or a specific correlation with the edge length of the triangular vacancy island of Figure 1.

The complexity of the corannulene monolayer phase is further confirmed by LEED patterns that we present in Figure 4. There, one can detect the coexistence of peaks belonging to two different periodicities. This is best appreciated in comparison with the Bragg's integer peaks from the substrate $(1 \times 1)$ lattice, as measured at $50 \mathrm{eV}$ and shown in Figure 4(a). Close to the screen center (zero order), peaks corresponding to the $1 / 3$ diffraction order (marked by squares in Figure 4) can be seen with an intensity comparable to the Bragg's integer peaks (marked by triangles in Figure 4). An additional set of peaks is marked by circles in Figure 4; these are rather weak and can only be detected around the Bragg's peaks close to fourth-integer position. At the lower electron kinetic energy of $30 \mathrm{eV}$, reported in Figure $4(\mathrm{~b})$, the $2 / 3$ order peaks also become visible, confirming the presence of a commensurate $(3 \times 3)$ symmetry pattern, corresponding to a periodicity of $\sim 8.6 \AA$. On the contrary, the fractional spots close to the position of fourth-integer peaks, neither develop a complete four-fold symmetry pattern ( 0.25 reciprocal lattice units, r.l.u., i.e. periodicity of $\sim 11.5 \AA$ ) nor display the emergence of spots of higher order beyond those surrounding the Bragg's integer peaks. At even lower kinetic energy (down to $21 \mathrm{eV}$ ), only the peaks belonging to the $1 / 3$ and $2 / 3$ diffraction orders can be detected (see Figure S4 of the Supporting Information).

The facts that i) the three-fold symmetry spots display an intensity and shape comparable with that of Bragg's integer peaks, ii) the $2 / 3$ order fractional spots display a strong intensity dependence on the electron energy, and iii) their intensity is enhanced at lower kinetic energy (larger escape depth) suggest that the three-fold pattern originates from the electron rich (high- $Z$ ) substrate atoms, pointing to a local substrate reconstruction. In comparison, the quasi four-fold spots display a fast degradation by irradiation (on a timescale of 1-2 min) when the electron kinetic energy exceeds $30 \mathrm{eV}$, their intensity is always weaker than that of the 


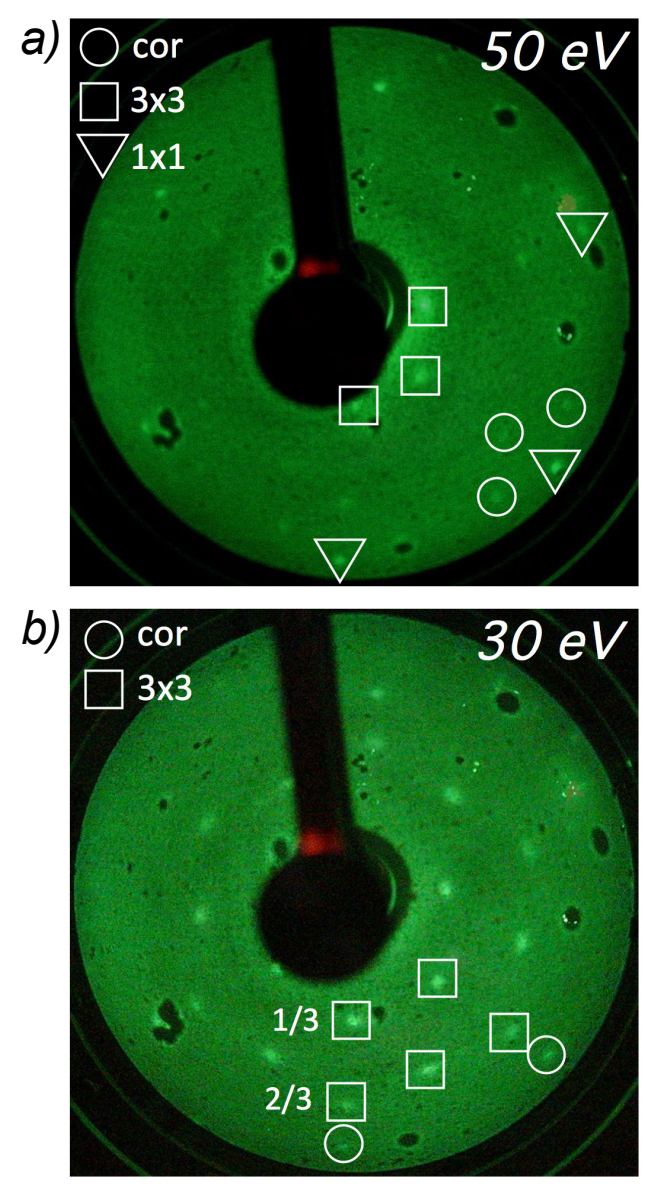

Figure 4: LEED patterns measured on a monolayer phase at RT. The substrate integer Bragg's peak, the three-fold reconstruction peaks and the corannulene (cor) superlattice peaks are marked by triangles, square and circles, respectively. Panel (a): pattern measured at an electron kinetic energy of $50 \mathrm{eV}$. Panel (b): same surface measured at $30 \mathrm{eV}$. Notice that LEED images are taken slightly off-normal incidence for better visualizing the diffraction orders; this geometry introduces a small asymmetric displacement of the diffracted spots from their nominal position. 
three-fold peaks, and they display a smooth fading with decreasing electron kinetic energy. These evidences indicate that the quasi-four-fold spots are associated with the low- $Z$ carbon atoms of the corannulene overlayer. Due to the large observed dynamics of corannulene, we hypothesize that the lack of higher orders of quasi-four-fold peaks originates by a distribution of slightly different intermolecular spacings due to the molecules that try to fit the $(1 \times 1)$ substrate lattice and the locally reconstructed $(3 \times 3)$ domains. In this regard, the quasi fourfold spots are at a distance of $0.27-0.28$ r.l.u. from the integer ones, which corresponds to a characteristic length of $10.4 \pm 0.2 \AA$, in perfect agreement with the average intermolecular spacing detected by STM.

The mismatch between the substrate long range order and the local arrangement of the molecular overlayer (either ordered or disordered) is not a new observation and was quantitatively measured for the monolayer phase of phthalocyanine on $\mathrm{Au}(110)$ by means of diffractive techniques with different probing depth. ${ }^{55,56}$ Finally, we did not find a clear evidence of additional spots to be associated with the long wavelength modulation of the molecular rows (see Figure 3), either yielding a higher order periodicity (for a commensurate superlattice) or a coincidence pattern (incommensurate overlayer). This is likely due to the already weak intensity of the quasi four-fold peaks stemming from corannulene, together with the poor quality of our LEED setup. The presence of a substrate reconstruction does not come fully unexpected when comparing corannulene with the case of $\mathrm{C}_{60}$, which gives rise to the formation of a single vacancy at the adsorption site. ${ }^{57}$ The adsorption of $\mathrm{C}_{60}$ at vacancies of different size is a general trend observed on fcc(111) metal surfaces and it is associated with a configuration where $\mathrm{C}_{60}$ sits on a bottom hexagon parallel to the surface. ${ }^{25}$ This observation further supports the hypothesis that some of the corannulene molecules may display a tilted orientation that brings one of the hexagons at the molecular rim close to the surface, hence driving a local $(3 \times 3)$ reconstruction of $\operatorname{Ag}(111)$. 


\section{Structure and Electronic Properties}

The long wavelength modulation (either commensurate or incommensurate) of the complex molecular phases along with the underlying substrate reconstruction presents a challenge for periodic DFT calculations. We therefore consider the adsorption processes of two strictly commensurate models: the compressed $(3 \times 3)$ phase and the relaxed $(4 \times 4)$ one to address the molecular layer periodicity that is intermediate between them.

We first considered adsorption with a perfectly commensurate $(3 \times 3)$ periodicity. Since the diameter of one molecule ( $8.6 \AA$ between the farthest $\mathrm{H}$ atoms) approximately equals the size of the $(3 \times 3)$ unit cell, only tilted configurations are possible in this phase.

We optimized the equilibrium configuration starting from several molecular orientations with different azimuthal angles $\phi$ (see Figure 5(a) for definition of $\phi$ ) and in-plane adsorption sites. We started structural optimizations with an initial tilt angle $\theta$ of $20^{\circ}$ (defined here as the angle made by the normal to the central pentagon with that of the substrate), corresponding to corannulene facing the substrate with a $\mathrm{C}$ hexagonal ring, similar to the adsorption geometry reported on the $\mathrm{Cu}(111)$ surface. ${ }^{8}$ We considered adsorption at the bridge (B), fcc hollow (F), hcp hollow (H), and top (T) fcc(111) high-symmetry sites (referred to the position of the closest $\mathrm{C}$ hexagon), and different azimuthal angles in steps of $15^{\circ}$ (with $\phi=0^{\circ}$ being forbidden by steric hindrance between the molecules), finally yielding eight inequivalent configurations (the four sites times two azimuths). The most stable configuration is visualized in Figure 5, and features the bottom hexagon at a surface bridge site B

at $2.93 \AA$ from the topmost metal plane, with $\phi=30^{\circ}, \theta=30.8^{\circ}$, and adsorption energy $E_{\text {ads }}=-1.53 \mathrm{eV}$. This increase of tilt angle upon optimization was observed for all eight configurations as a result of the short intermolecular distance. Remarkably, all cases lie in a narrow energy range (see the Supporting Information), with both hollow sites only $0.02 \mathrm{eV}$ higher in energy than the $\mathrm{B}$ one.

The $(3 \times 3)$ configuration is characterized by a small electron transfer from the molecule to the substrate, consistent with the relatively low electron affinity of corannulene (about 


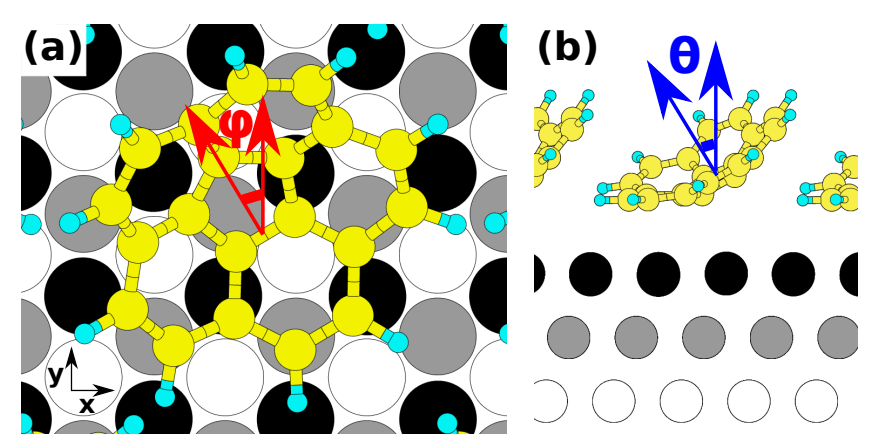

Figure 5: (a) Top view and (b) side view of the most stable $(3 \times 3)$ configuration (bridge site B). Color codes as follows:- yellow: carbon, blue: hydrogen, black: first layer, grey: second layer and white: third layer Ag atoms). The azimuthal angle $\phi$ is marked in red in panel (a) and is defined as the angle between the substrate [011] direction $(y)$ and the apothem of the hexagon facing the surface. The tilt angle $\theta$ is marked in blue in panel (b) and is defined as the angle between the surface normal and the normal to the pentagon ring.

$\left.0.5 \pm 0.1 \mathrm{eV}^{58}\right)$. By using the Löwdin charge partitioning scheme, ${ }^{59}$ we find that the molecule donates $\sim 0.3$ electrons to $\mathrm{Ag}$, a result practically identical to the one of corannulene $/ \mathrm{Cu}(111)$, where the molecules also adopt a tilted adsorption configuration lying on a hexagon. ${ }^{60}$ The corresponding charge rearrangement is visualized in Figure 6(a), where we report the adsorption charge density evaluated by subtracting both the molecular and the substrate electronic densities from the total electronic density of the combined system. The blue/red regions depict the depletion/accumulation of electronic density. This highlights that electron transfer mostly involves the part of the molecule closer to the Ag surface. Note the relatively small isovalue of $\pm 0.002 e / \AA^{3}$ used here as no charge displacements were visible at larger isovalues such as the ones used to describe other adsorbed systems characterized by more significant electron transfer ${ }^{61}$ or chemical bonds. ${ }^{48}$ The simulated STM image for corannulene adsorbed at the bridge sites of $\mathrm{Ag}(111)$ is depicted in Figure 6(b). As expected, the molecules display a clear asymmetric contrast with a larger intensity in correspondence of the more protruding atoms. These simulations reproduce with remarkable accuracy the STM intramolecular features measured for molecules trapped within a triangular vacancy island (Figure 1), although the measured intermolecular spacing is larger than that of the calculated $(3 \times 3)$ phase. However, we remark that this is a very specific case where the 
interaction with the surrounding step edges appears to freeze the molecules forcing them to adopt a unique molecular tilt and azimuth.

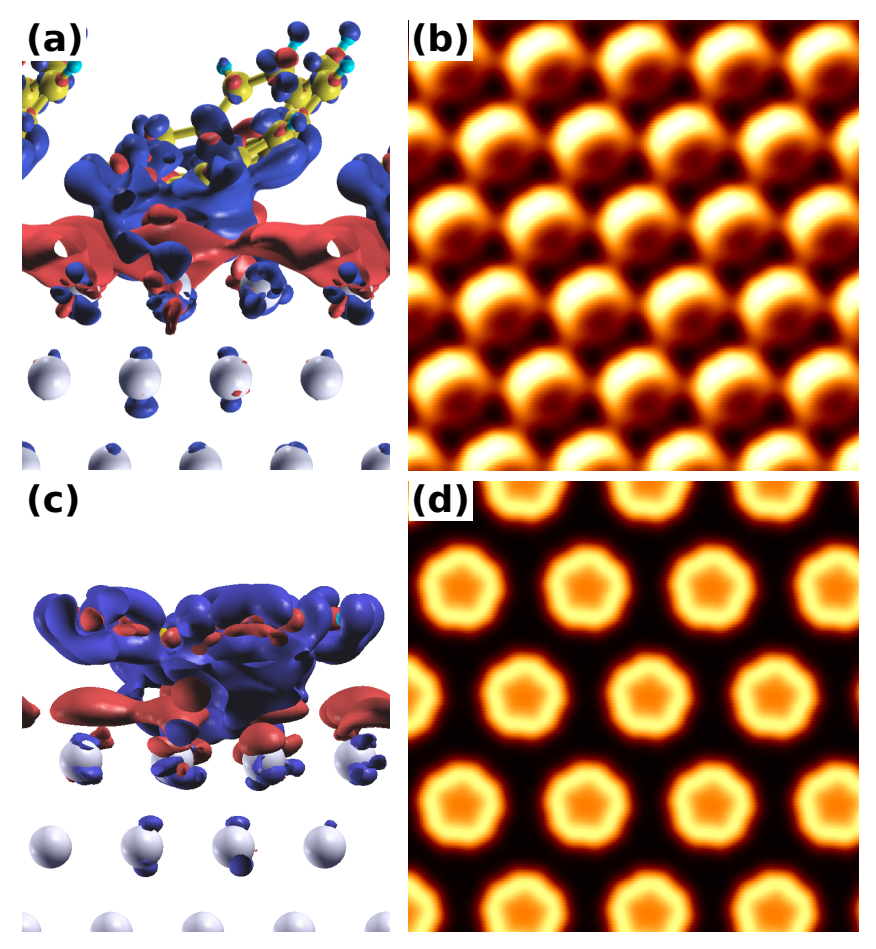

Figure 6: Calculated charge density and STM for the most stable configurations in the $(3 \times 3)$ (panels a,b) and $(4 \times 4)$ (panels c,d) phases. $(\mathrm{a}, \mathrm{c})$ Side view of adsorption charge displacement, $\rho_{\text {Corannulene } / \operatorname{Ag}(111)}-\rho_{\text {Corannulene }}-\rho_{\operatorname{Ag}(111)}$. Blue: electronic charge depletion and red: electronic charge accumulation, with isovalue of $\pm 0.002 e / \AA^{3}$. (b,d) Simulated STM image at the bridge site for a tunneling voltage of $+1 \mathrm{~V}$. The apparent height (as measured from the surface plane) is reported, corresponding to an isovalue of the integrated local density of states equal to $1 \times 10^{-5} \mathrm{e} / \AA^{3}$.

We then consider the case of corannulene in a $(4 \times 4)$ cell, where the larger intermolecular separation allows for any value of molecular azimuth and tilt without any steric constraint. We selected initial values of tilt angle $\theta=0^{\circ}, 10^{\circ}$, and $20^{\circ}$, corresponding to the case of corannulene facing the surface with the central pentagon, a pentagon $\mathrm{C}-\mathrm{C}$ bond, and a hexagon, respectively. These molecular orientations were taken as reference and placed on the T, H, F, and B sites, with values of azimuthal angle $\phi$ from $0^{\circ}$ to $105^{\circ}$ in steps of $15^{\circ}$ resulting in 96 total initial geometries. See the Supporting Information for more details. In all the cases, the molecule ended up in a flat-lying configuration ( $\theta$ no larger than $2^{\circ}$ ). Among them, the configuration centered on a fcc hollow site was slightly preferred, attaining 
an adsorption energy of $-1.66 \mathrm{eV}$ with $\phi=30^{\circ}$ and the central pentagon at $2.77 \AA$ from the first Ag layer. The dependence on the azimuthal orientation and adsorption site is very mild (the energy increases by at most $0.01 \mathrm{eV}$ when changing $\phi$ from that configuration, or for adsorption at an hcp or bridge site) that is indicative of the absence of significant localization of the interaction with the three-fold symmetry fcc site and adjacent molecules, and points to a very shallow potential energy surface. For a better understanding of the adsorption mechanism, we repeated the calculations for the flat-lying molecule in the $(4 \times 4)$ unit cell, but adopting the Perdew-Burke-Ernzerhof GGA exchange-correlation functional, ${ }^{62}$ that does not reproduce dispersion forces. In this case, the computed adsorption energy is only $0.15 \mathrm{eV}$, which indicates that molecules are simply physisorbed and the coupling to the substrate is essentially driven by van der Waal's forces.

The increase of adsorption strength of individual molecules in the less dense phase is not surprising, when the adsorption is simply driven by van der Waal's interaction with the substrate. However, the surface energy balance can still favour the most dense phase upon weighing the adsorption energy for the film density. ${ }^{63}$ In the present case, the $(3 \times 3)$-tilted phase results to be favoured by $9 \mathrm{meV} / \AA^{2}$ with respect to the $(4 \times 4)$-flat one. As a final remark on the tilt angle, the flat-lying geometry of $(4 \times 4)$ molecules demonstrates that the substantial value of $\theta$ in the denser phase is a result of intermolecular interactions. In this respect, it may be interesting to mention that a large tilt angle for isolated molecules as well could instead be induced by the addition of aromatic rings at the rim of corannulene, as shown by a recent study of terphenylcorannulene on $\mathrm{Cu}(111) \cdot{ }^{64}$

Also in the $(4 \times 4)$ case, corannulene was found to charge positively $(\sim 0.4$ electrons transfered to the substrate) with an electron displacement shown in Figure 6(c). When looking at the STM simulations for the $(4 \times 4)$ model, shown in Figure $6(d)$, corannulene displays a clear intensity enhancement at the molecular rim, which is qualitatively consistent with the high resolution images taken at the smaller scale, see Figure 2(a). On the contrary, simulations show in Figure 6(d) a pentagonal shape of the molecular edges that apparently 
contrasts with the rounded, although sharp, edge of our best resolved measurements. We have seen that the measured molecular contrast results from dynamical effects strongly influenced by the tip interaction: in this respect, the hypothesis of a fast spinning dynamics at room temperature is fully consistent with the predicted shallowness of the adsorption energy dependence on the azimuthal orientation.

As we discussed in the previous section, our experimental results and the comparison to the literature do not suggest the coexistence of molecule exhibiting the downward concavity (bowl-down). We additionally remark that the bowl-up geometry is the only one allowing corannulene to be accommodated into a compressed phase by a relatively inexpensive change of the molecular tilt with respect to a relaxed $(4 \times 4)$ phase, as also observed for corannulene on $\mathrm{Cu}(111) .{ }^{8}$ Additional calculations performed for bowl-down molecules further support this interpretation. When considering the relaxed phase, all $\mathrm{H}$ atoms of a bowl-down molecule reside close to the substrate at the expenses of the $\pi$ electron system being instead farther, resulting in practically the same adsorption energy as for the bowl-up orientation (the latter being favoured by only $0.01 \mathrm{eV}$ ). It may be interesting to mention that a bowl-down geometry is computed to be more favorable for corannulene radicals resulting from debromination of Br-corannulene on $\mathrm{Ag}(110)$, where this allows bonding to surface Ag adatoms. ${ }^{65}$ When taking the compressed phase with tilted adsorbates, bowl-down molecules need to raise a fraction of

their H atoms over the substrate, whereas the bowl-up case can pass almost continuously from on-pentagon to on-hexagon adsorption with comparatively smaller changes in the distance of the $\pi$ electron system from Ag: correspondingly, we computed a lower energetic stability of bowl-down than bowl-up molecules in the $(3 \times 3)$ phase by $0.42 \mathrm{eV}$. Hereafter, we will only consider molecules oriented in a bowl-up geometry.

\section{Core level photoemission}

The measured and computed XPS spectra of the adsorbed corannulene are shown in Figure 7. The experimental line (blue solid line) is characterized by a single feature centered at a 
binding energy (BE) of about $285.1 \mathrm{eV}$. The shallow component at $3.5-4.0 \mathrm{eV}$ higher $\mathrm{BE}$ corresponds to a shake-up satellite associated with the photoelectron energy loss of exciting a HOMO-LUMO gap transition, ${ }^{66}$ where the gap of free corannulene was calculated to be $4.3 \mathrm{eV} .{ }^{67}$ On the main $\mathrm{C}$ 1s peak, the individual contributions by the inequivalent carbon sites are unresolved and we did not attempt a fitting of the measured spectrum in terms of their contributions, which are instead addressed theoretically.

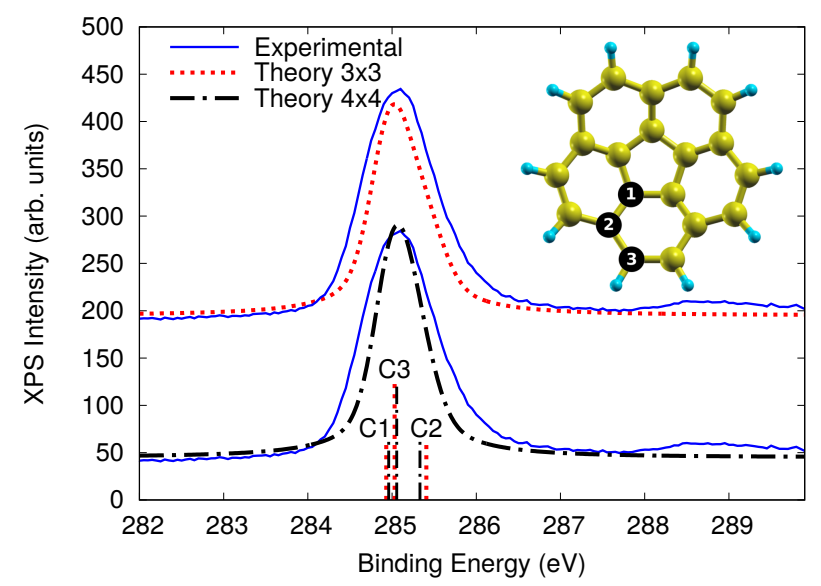

Figure 7: XPS spectrum of corannulene measured for $1 \mathrm{ML}$ on $\mathrm{Ag}(111)$ with photon energy $h \nu=515 \mathrm{eV}$ (blue solid line), compared to the simulated ones in the $(3 \times 3)$ (red dotted line, stacked for clarity) and $(4 \times 4)$ phase (black dash-dotted line). Vertical sticks show the average contributions from the three groups of $\mathrm{C}$ atoms as numbered in the inset. The theoretical spectrum is obtained by broadening the individual $\mathrm{C}$ contributions with a pseudoVoigt profile (50\% Gaussian plus 50\% Lorentzian both having full width at half maximum of $0.5 \mathrm{eV}$ ), and have been aligned to $285.10 \mathrm{eV}$.

The simulated C 1s spectrum of 1 ML corannulene on $\mathrm{Ag}(111)$, shown in Figure 7 as a red dotted line, is obtained by evaluating the core level shifts (CLS) among the binding energy (BE) of the inequivalent $\mathrm{C}$ atoms and the center of the line; ${ }^{46}$ the latter is not accessible by the pseudopotential method and is aligned to the experiments. A pseudo-Voigt profile is then assigned to each atom and summed to get the total spectrum. The overall result is in good agreement with the experimental findings and can be analyzed in terms of the atomic contributions. Average values of the binding energies are calculated for each of the three distinct types of $\mathrm{C}$ atoms, labeled $\mathrm{C} 1, \mathrm{C} 2$, and $\mathrm{C} 3$, and grouped in order of increasing distance from the center of the molecule (see Figure 7, inset). The results are marked as 
sticks in Figure 7 and given in Table 1. We can identify the C1 (C2) group as the one at lower (higher) BE, whereas the most abundant C3 species contributes intermediate values. The lower binding energy of the $\mathrm{C} 1$ atom with respect to $\mathrm{C} 2$ is consistent with the predictions for fullerenes. ${ }^{68,69}$ In fact, the relative C1 energy is further lowered when considering the gas phase molecule (presented in the Supporting Information), which shows the same relative order of the binding energies $\left(\mathrm{BE}_{\mathrm{C} 1}<\mathrm{BE}_{\mathrm{C} 3}<\mathrm{BE}_{\mathrm{C} 2}\right)$.

Table 1: Calculated C 1s core level shifts of adsorbed corannulene in the $(3 \times 3)$ and $(4 \times 4)$ configurations. For each group (C1, C2, C3, see Figure 7 for the definition), we provide the average value of the CLS of individual C atoms, as weighted by their multiplicity.

\begin{tabular}{ccc} 
Species & $(3 \times 3)$ CLS $(\mathrm{eV})$ & $(4 \times 4)$ CLS $(\mathrm{eV})$ \\
\hline C1 & -0.169 & -0.141 \\
C2 & +0.307 & +0.231 \\
C3 & -0.069 & -0.045 \\
\hline C2-C3 & +0.376 & +0.276
\end{tabular}

Looking to the CLS of individual atoms, atoms of the same group are practically equivalent in the $(4 \times 4)$ phase, while the molecular tilting in the $(3 \times 3)$ phase introduces an additional variation due to the change of atomic distance from the surface. The corresponding CLSs are reported in Table 2, together with the height over the surface plane, $z_{\mathrm{C}}$. This quantity can experience significant variations as a result of molecular tilt, especially for the C3 species where $z_{\mathrm{C}}$ ranges from $2.81 \AA$ to $5.86 \AA$. A net trend is observed from the data, with the larger distance from the surface resulting in a higher binding energy, corresponding to a less effective surface screening. The spread of the individual CLS of carbon atoms within a group in the $(3 \times 3)$ configuration results in a broader convoluted peak and slightly more pronounced skewness towards high binding energy with respect to the $(4 \times 4)$ phase. In this regard, the experimental XPS spectrum does not allow for a thorough deconvolution to be quantitatively compared with the calculated CLSs, hence the possible contribution from tilted molecules cannot be discriminated solely on the basis of the XPS width and skewness. 
Table 2: Calculated C 1s core level shifts, CLS, and distance from the surface $z_{\mathbf{C}}$ of the inequivalent carbon atoms, each one occurring with a multiplicity $n_{\mathbf{C}}$ in the tilted $(3 \times 3)$ configuration.

\begin{tabular}{cccc} 
Species & CLS $(\mathrm{eV})$ & $z_{\mathrm{C}}(\AA)$ & $n_{\mathrm{C}}$ \\
\hline C1 & -0.201 & 3.00 & 2 \\
& -0.167 & 3.69 & 2 \\
& -0.110 & 4.11 & 1 \\
\hline C2 & +0.285 & 2.98 & 2 \\
& +0.275 & 4.37 & 2 \\
& +0.414 & 5.27 & 1 \\
\hline C3 & -0.147 & 2.81 & 2 \\
& -0.140 & 3.41 & 2 \\
& -0.104 & 4.08 & 2 \\
& +0.038 & 5.43 & 2 \\
& +0.007 & 5.86 & 2
\end{tabular}

\section{NEXAFS}

The NEXAFS spectra for $1 \mathrm{ML}$ of corannulene/ $\mathrm{Ag}(111)$ are reported in Figure 8 , as measured in p- and s-polarization scattering geometries. The corresponding theoretical spectra are calculated for the molecules adsorbed on the $\mathrm{Ag}(111)$ slab in the $(3 \times 3)$ and $(4 \times 4)$ periodicities, with the electric field perpendicular to the surface plane (p-polarization) and parallel to it (s-polarization, averaged over the surface azimuths). We obtained a good agreement in the peak sequence and energetic position between the experimental and calculated resonances, which allows us to interpret the measurements by virtue of the numerical analysis. Relative intensities differ to some extent, especially for energies $\approx 285 \mathrm{eV}$, where the simulated intensities are clearly underestimated. In this respect, we recall that the halfcore-hole approximation that is taken here produces lower intensities than other core-hole potentials (in particular, the full-core-hole one) and that the performance of the $\mathrm{HCH}$ over the FCH is in general assessed a posteriori: For example, the HCH was identified as a better approach for water ${ }^{70}$ and was used successfully for polyaromatics of comparable size, ${ }^{37,46}$ but the FCH was deemed necessary for the description of XAS in $\mathrm{C}_{60}$ and $\mathrm{C}_{70} \cdot{ }^{71}$ The current case is somewhat intermediate, as we demonstrate by additional calculations (presented in the 
Supporting Information) where we compare the FCH and $\mathrm{HCH}$ spectra of free corannulene. The FCH overly amplifies the LUMO NEXAFS resonance to an extent that worsens the comparison to experiments, hence providing both a rationale for the observed discrepancies of the $\mathrm{HCH}$ and a confirmation of its appropriateness for corannulene.
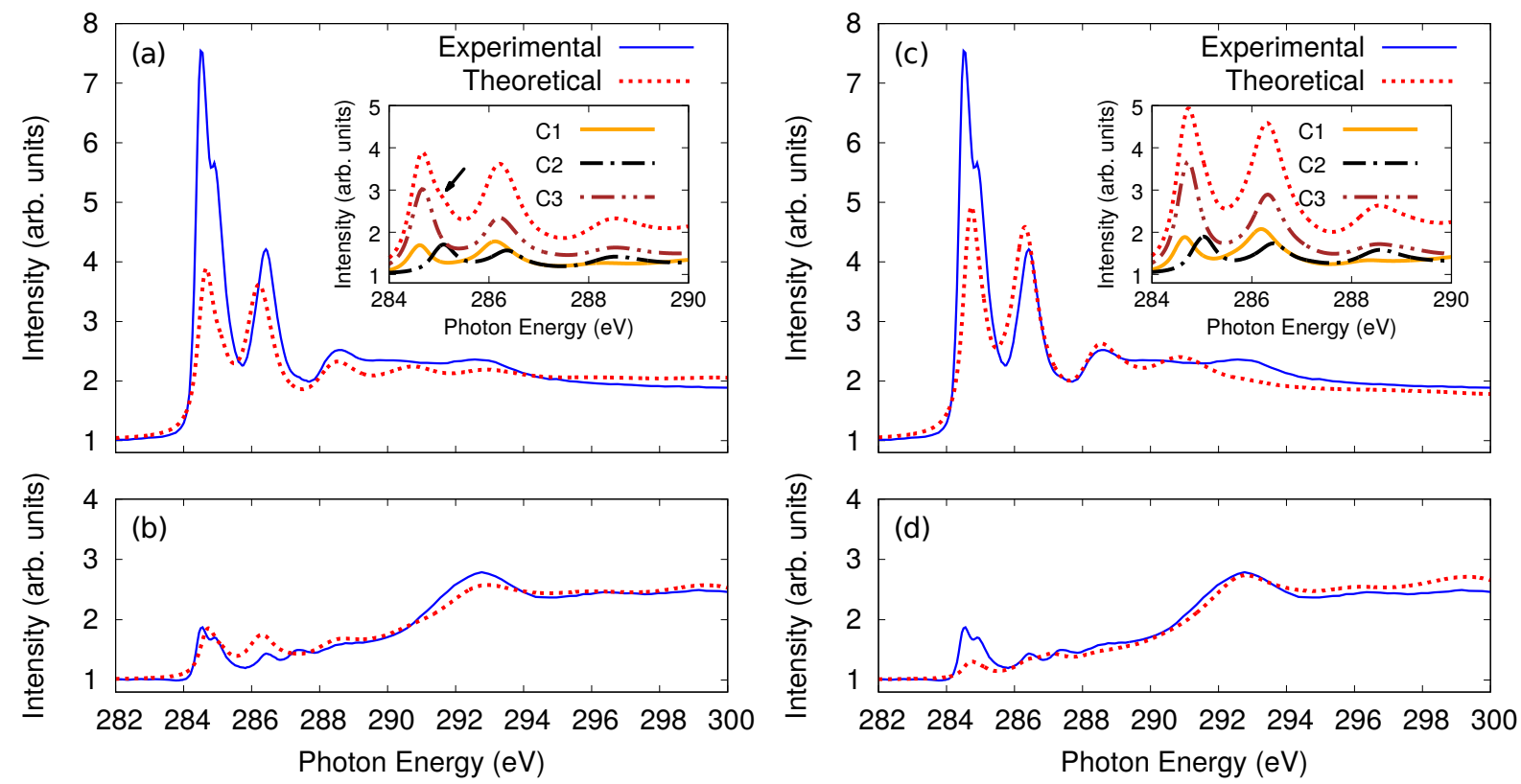

Figure 8: Experimental and simulated NEXAFS spectra of 1 ML corannulene/ $\operatorname{Ag}(111)$ for the $(3 \times 3)$ and $(4 \times 4)$ configurations ( $\mathrm{a}-\mathrm{b}$ and $\mathrm{c}-\mathrm{d}$ panels, respectively), as measured in $\mathrm{p}$ - and s-polarization (a-c and b-d panels, respectively). The insets of the panels a and c report the contributions by the three groups of $\mathrm{C}$ species defined in Figure 7. The theoretical spectra are shifted by $284.55 \mathrm{eV}$ to align to the experimental LUMO features, and rescaled to match the NEXAFS intensity in the pre-edge region and far above the ionization threshold.

The measured spectrum in p-polarization shows the first most prominent transition at about $284.55 \mathrm{eV}$ with an additional sharp state at slightly higher energy $(\sim 284.9 \mathrm{eV})$. A theoretical analysis in terms of individual contributions to the calculated spectrum shows that both features originate from transitions to the $\pi^{*}$ LUMO state, where the splitting is simply due to the different CLSs of the different carbon species (initial state effect). In fact, the atomically resolved NEXAFS simulations reported in the insets of Figure 8 show that the first peak of the $284.5 \mathrm{eV}$ feature is due mostly to $\mathrm{C} 3$ atoms and, to a smaller extent, C1 type. The other $(\mathrm{C} 2)$ atoms yield a second contribution at higher energy (see arrow in 
the inset of Figure 8a), in agreement with the computed CLSs and experiments. Again, the presence of tilted molecules is expected to be at the origin of the enhanced splitting of the LUMO resonances that we compute in the denser phase. From comparison with NEXAFS simulations for a free molecule (see the Supporting Information), we can further identify the resonance at $286.4 \mathrm{eV}$ with an overlap of the transitions to $\mathrm{LUMO}+2$ and $\mathrm{LUMO}+3$ states of corannulene, whereas transitions to the LUMO+1 are absent because of vanishing transition dipole. A third weaker and broader structure is observed close to the ionization threshold at $\sim 288.6 \mathrm{eV}$ both in the experiments and in the simulations, corresponding to higher molecular orbitals mostly localized on C2 atoms.

Focusing now on the spectrum in s-polarization, this shows a relatively large residual intensity of the first $\pi^{*}$ resonance at $\sim 284.5 \mathrm{eV}$ indicating a tilt of these states with respect to the surface as a combined result of intrinsic molecular convexity and possible adsorption induced molecular tilt. Apart from LUMOs intensities that will be discussed in more detail hereafter, there is a remarkable agreement between the experimental and simulated spectra for adsorbed molecules. The only $\sigma$-symmetry resonance appearing below the ionization threshold in s-polarization is found at $\sim 287.3 \mathrm{eV}$, which also comparing to the simulations for a free molecule in the Supporting Information can be attributed to the $\mathrm{C}-\mathrm{H} \sigma^{*}$ bonds of $\mathrm{H}$ atoms attached to $\mathrm{C} 3$ carbon atoms. Finally, in the region of the continuum resonances, we observe three broad $\sigma$-symmetry resonances (at about $292.8,296.5$ and $299.5 \mathrm{eV}$ ). The comparison with the simulated spectra highlights the adequacy of the theoretical approach, where the peaks are reproduced with high accuracy both in their energy position and relative intensities.

Now we are in a position to evaluate the molecular tilt angle which can be obtained from the ratio of the residual $\pi^{*}$ resonances in s- and p-polarizations by means of Stöhr's analysis. ${ }^{72}$ Experimentally, the intensity ratio of the $\pi^{*}$-symmetry resonances in s- and p- polarization $\left(I_{s} / I_{p} \sim 0.14\right.$ for transitions in the $284-285.5 \mathrm{eV}$ range) allows us to estimate an average molecular tilt angle $\gamma \sim 28 \pm 2^{\circ}$ according to the simple relationship $I_{s} / I_{p}=1 / 2 \tan ^{2} \gamma$. We 
highlight that this angle is conceptually different from the true molecular tilt angle, $\theta$, and their identification is strictly applicable only in highly symmetric cases (e.g., linear molecules or flat polyaromatics). In corannulene, the bowl-shaped geometry results in a curvature of the molecular orbitals. As an example, the ground state LUMO for a free molecule is depicted in the inset of Figure 9. One can observe that the orbital has the symmetry of a $2 p$ state around $\mathrm{C}$ atoms, which is however oriented at an angle $\beta$ from the molecular axis orthogonal to the central pentagon ( $z$ in the figure). This angle is determined as $\beta=\arctan \left(\sqrt{P_{x y} / P_{z}}\right)$ where $P_{\nu}=\left|\left\langle\Psi_{\mathrm{LUMO}} \mid 2 p_{\nu}\right\rangle\right|^{2}$ are the projections of the LUMO wavefunction $\Psi_{\mathrm{LUMO}}$ on the $2 p_{\nu}$ states of the considered atom $(\nu=x, y, z)$. The results, tabulated within Figure 9 , show that the LUMO dipole on the $\mathrm{C} 2$ atoms is oriented at the largest value of $\beta=25^{\circ}$ from the $z$-axis, but the LUMO deviates by $\approx 20^{\circ}$ from the $z$-axis also at $\mathrm{C} 1$ and $\mathrm{C} 3$.

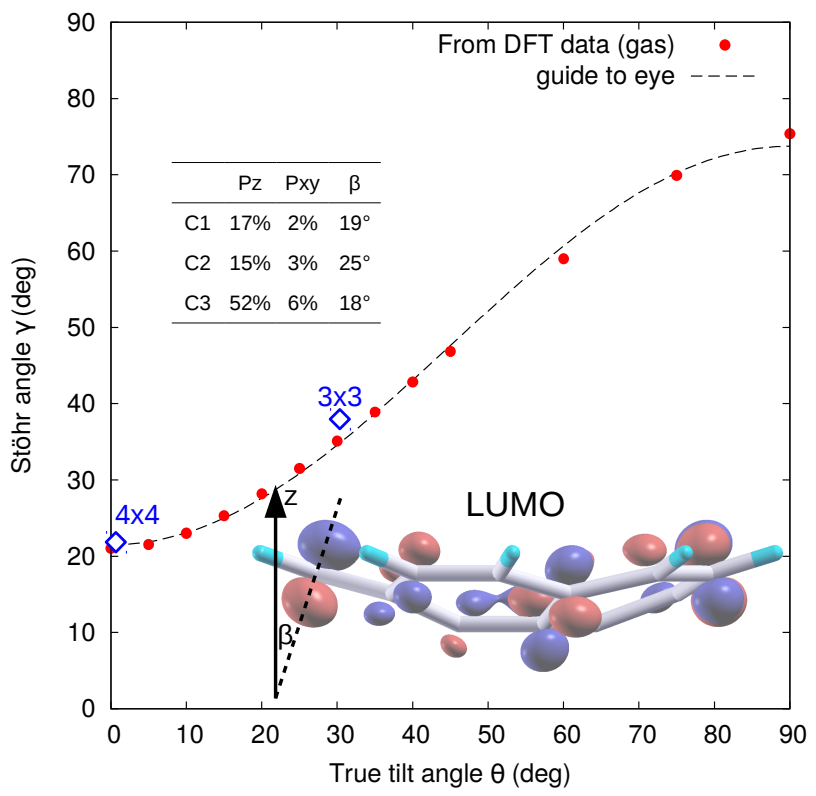

Figure 9: Dependence of the average tilt angle $\gamma$ determined by Stöhr analysis for gas phase corannulene on the angle $\theta$ between the molecular axis and the electric field in p-polarization. Inset: side view of the LUMO of gas phase corannulene and schematics of the local slanting of the $\pi$ state (here shown at a peripheral C3 atom). The table reports the amplitude of the LUMO wavefunction over the $2 p$ components, aligned along the molecular axis $\left(P_{z}\right)$ and perpendicular to it $\left(P_{x y}\right)$, and the effective angle $\beta$ formed by the LUMO with the $z$-axis at the various $\mathrm{C}$ sites.

It follows that in the present case, the angle $\gamma$ extracted from the common application of 
the Stöhr analysis does not correspond to the true molecular tilt angle $\theta$, and the relation $\gamma(\theta)$ depends on the specific curvature of corannulene. We analyze this effect by considering the theoretical NEXAFS simulations for a free corannulene molecule (presented in the Supporting Information) as a function of its tilt angle $\theta$ with respect to a reference plane. We then evaluated the corresponding angle drawn from the Stöhr's analysis applied to the LUMO resonances in the 284.0-285.5 eV energy range (a procedure also adopted by other authors ${ }^{73}$ ). The results are presented in Figure 9), where one sees that a minimum angle of $\gamma=21^{\circ}$ is expected even for a flat-lying molecule $\left(\theta=0^{\circ}\right)$, as purely resulting from the curved molecular shape. At larger molecular tilt angles $\theta$, the computed values could be fit by a cosine expression, whose maximum value just reaches $\sim 75^{\circ}$ for an on-edge standing molecule. It could be noted that the experimental angle $\left(\gamma_{\text {exp }} \sim 28 \pm 2^{\circ}\right)$ is intermediate between the calculated values for an on-pentagon $(4 \times 4)$ phase $\left(\theta_{4 x 4} \sim 0^{\circ}, \gamma_{4 x 4} \sim 22^{\circ}\right.$, and an on-hexagon $(3 \times 3)$ one $\left(\theta_{3 x 3} \sim 30^{\circ}, \gamma_{3 x 3} \sim 38^{\circ}\right)$ (minor differences with respect to the values quoted above originate from the use of the adsorbed instead of gas phase simulations). From comparison with the STM images, we can consistently conclude that corannulene molecules display a tilt angle in between these two extreme values, which together with varying azimuthal angle gives rise to the observed long wavelength modulation of the contrast along the molecular rows (Figure 3). In this regard, we remark that tilting around the two azimuths yields the same result, due to the molecular symmetry.

\section{Conclusions}

Corannulene was deposited on $\mathrm{Ag}(111)$ and studied by experimental STM, X-ray photoemission and absorption spectroscopies, and the results compared with those of DFT calculations. The molecules display a very high mobility at room temperature with larger residence time at steps and defects. Stable and extended molecular domains are only observed close to the completion of the first layer, where molecules self assemble into a close-compact ordered 
phase made only of bowl-up oriented molecules with an average intermolecular spacing of $\sim 10.5 \AA$. We occasionally observed a long scale weak modulation of the STM contrast along the molecular rows, which we attribute to the mismatch with the $\mathrm{Ag}(111)$ lattice, although we did not observe a specific superlattice periodicity. In particular, the molecular appearance (and arrangement) at both low and monolayer coverage was found to be susceptible to the tip interaction either due to a change of the tip state or of the tip residence time (scanning speed). Low energy electron diffraction (LEED) measurements show the additional presence of a three-fold diffraction pattern $(\sim 8.6 \AA$ periodicity), which we attribute to a local reconstruction of substrate regions that, although spatially separated, are correlated over a long range. In order to address the electronic and structural properties of this molecular ensemble, we modelled corannulene in two commensurate phases: a three-fold (compressed) phase, where the molecule is at equilibrium by adsorption on a hexagon (tilted geometry), and a four-fold (relaxed) phase $(\sim 11.5 \AA$ periodicity), where the molecule is at equilibrium by adsorption on the central pentagon (bowl-up). For a better understanding of the molecular orientation, we performed a thorough analysis of the NEXAFS dichroism in p- and s-polarization taking into account the molecular curvature. The corresponding average tilt angle is found to be intermediate between those of the two limiting phases. All the structural and spectroscopic measurements are intermediate between the simulated properties of the molecules in the two limiting geometries. We suggest that the long wavelength modulation of the molecular rows corresponds to a sequence of molecules displaying a spatial modulation of the tilt angle between the values of the three- and four-fold phases.

\section{Supporting Information}

Supporting Information. STM topography of the clean sample. STM topography in the submonolayer range. Restructuring of edges of small vacancy islands as seen by STM. Additional LEED measurements. Details on the DFT energetics of $(3 \times 3)$ and $(4 \times 4)$ 
phases. XPS and NEXAFS simulations for gas phase corannulene. Analysis of different core-hole potentials for NEXAFS spectra. Initial/final-state effects in simulated NEXAFS. The Supporting Information is available free of charge on the ACS Publications website at DOI: 10.1021/acs.jpcc.XXXXXX.

\section{Acknowledgement}

The simulations were performed at the super computers in CINECA, Italy. He Lin is grateful to Pirelli company for funding his Ph.D. scholarship. Carla Bittencourt is supported by the Belgian Fund for Scientific Research (FSR-FNRS) as Research associate; "Chemographene" $\left(\mathrm{N}^{\circ} 2.4577 .11\right)$. Luca Floreano acknowledges partial financial support from the Consiglio Nazionale delle Ricerche - Istituto Nazionale di Fisica Nucleare (PROGETTO PREMIALE 2012, EOS). This work was performed under the European Spectroscopy Facility (ETSF) ${ }^{74}$ user project 544 .

\section{References}

(1) Sygula, A.; Fronczek, F. R.; Sygula, R.; Rabideau, P. W.; Olmstead, M. M. A Double Concave Hydrocarbon Buckycatcher. J. Am. Chem. Soc. 2007, 129, 3842.

(2) Xiao, W.; Passerone, D.; Ruffieux, P.; Aït-Mansour, K.; Gröning, O.; Tosatti, E.; Siegel, J. S.; Fasel, R. C $60 /$ Corannulene on $\mathrm{Cu}(110)$ : A Surface-Supported Bistable Buckybowl-Buckyball Host-Guest System. J. Am. Chem. Soc. 2008, 130, 4767-4771.

(3) Filatov, A. S.; Ferguson, M. V.; Spisak, S. N.; Li, B.; Campana, C. F.; Petrukhina, M. A. Bowl-Shaped Polyarenes as Concave-Convex Shape Complementary Hosts for $\mathrm{C}_{60^{-}}$and $\mathrm{C}_{70}$-Fullerenes. Cryst. Growth Des. 2014, 14, 756-762.

(4) Vilmercati, P.; Castellarin-Cudia, C.; Gebauer, R.; Ghosh, P.; Lizzit, S.; Petaccia, L.; Cepek, C.; Larciprete, R.; Verdini, A.; Floreano, L. et al. Mesoscopic Donor-Acceptor 
Multilayer by Ultrahigh-Vacuum Codeposition of Zn-Tetraphenyl-Porphyrin and $\mathrm{C}_{70}$. J. Am. Chem. Soc. 2009, 131, 644-652.

(5) Schiros, T.; Kladnik, G.; Prezzi, D.; Ferretti, A.; Olivieri, G.; Cossaro, A.; Floreano, L.; Verdini, A.; Schenck, C.; Cox, M. et al. Donor-Acceptor Shape Matching Drives Performance in Photovoltaics. Adv. Energy Mater. 2013, 3, 894-902.

(6) Xiao, W.; Ernst, K.-H.; Palotas, K.; Zhang, Y.; Bruyer, E.; Peng, L.; Greber, T.; Hofer, W.; Scott, L.; Fasel, R. Microscopic Origin of Chiral Shape Induction in Achiral Crystals. Nat. Chem. 2016, 8, 326-330.

(7) Rui, G.; Jialin, Z.; Songtao, Z.; Xiaojiang, Y.; Shu, Z.; Shuo, S.; Zhenyu, L.; Wei, C. LT-STM Investigation of the Self-Assembled $\mathrm{F}_{16} \mathrm{CuPc}_{\mathrm{P}}$-Corannulene Binary System on $\operatorname{Ag}(111)$ and Graphite Surfaces. Acta Phys. -Chim. Sin. 2017, 33, 627-632.

(8) Merz, L.; Parschau, M.; Zoppi, L.; Baldridge, K. K.; Siegel, J. S.; Ernst, K.-H. Reversible Phase Transitions in a Buckybowl Monolayer. Angew. Chem. Int. Ed. 2009, 48, 1966-1969.

(9) Parschau, M.; Fasel, R.; Ernst, K.-H.; Gröning, O.; Brandenberger, L.; Schillinger, R.; Greber, T.; Seitsonen, A. P.; Wu, Y.-T.; Siegel, J. S. Buckybowls on Metal Surfaces: Symmetry Mismatch and Enantiomorphism of Corannulene on $\mathrm{Cu}(110)$. Angew. Chem. Int. Ed. 2007, 46, 8258-8261.

(10) Barth, W. E.; Lawton, R. G. Dibenzo[ghi,mno]fluoranthene. J. Am. Chem. Soc. 1966, $88,380-381$.

(11) Wu, Y.-T.; Siegel, J. S. Aromatic Molecular-Bowl Hydrocarbons: Synthetic Derivatives, Their Structures, and Physical Properties. Chem. Rev. 2006, 106, 4843-4867.

(12) Ayalon, A.; Rabinovitz, M.; Cheng, P.-C.; Scott, L. T. Corannulene Tetraanion: A 
Novel Species with Concentric Anionic Rings. Angew. Chem. Int. Ed. 1992, 31, 16361637.

(13) Baldridge, K. K.; Siegel, J. S. Corannulene-Based Fullerene Fragments $\mathrm{C}_{20} \mathrm{H}_{10}-\mathrm{C}_{50} \mathrm{H}_{10}$ : When Does a Buckybowl Become a Buckytube? Theor. Chem. Acc. 1997, 97, 67-71.

(14) Butterfield, A. M.; Gilomen, B.; Siegel, J. S. Kilogram-Scale Production of Corannulene. Org. Process Res. Dev. 2012, 16, 664-676.

(15) Kuvychko, I. V.; Spisak, S. N.; Chen, Y.-S.; Popov, A. A.; Petrukhina, M. A.; Strauss, S. H.; Boltalina, O. V. A Buckybowl With a Lot of Potential: $\mathrm{C}_{5}-\mathrm{C}_{20} \mathrm{H}_{5}\left(\mathrm{CF}_{3}\right)_{5}$. Angew. Chem. Int. Ed. 2012, 51, 4939-4942.

(16) Schmidt, B. M.; Seki, S.; Topolinski, B.; Ohkubo, K.; Fukuzumi, S.; Sakurai, H.; Lentz, D. Electronic Properties of Trifluoromethylated Corannulenes. Angew. Chem. Int. Ed. 2012, 51, 11385-11388.

(17) Kuvychko, I. V.; Dubceac, C.; Deng, S. H. M.; Wang, X.-B.; Granovsky, A. A.; Popov, A. A.; Petrukhina, M. A.; Strauss, S. H.; Boltalina, O. V. $\mathrm{C}_{20} \mathrm{H}_{4}\left(\mathrm{C}_{4} \mathrm{~F}_{8}\right)_{3}$ : A Fluorine-Containing Annulated Corannulene that Is a Better Electron Acceptor Than $\mathrm{C}_{60}$. Angew. Chem. Int. Ed. 2013, 52, 7505-7508.

(18) Schmidt, B. M.; Topolinski, B.; Yamada, M.; Higashibayashi, S.; Shionoya, M.; Sakurai, H.; Lentz, D. Fluorinated and Trifluoromethylated Corannulenes. Chem. Eur. J. 2013, 19, 13872-13880.

(19) San, L. K.; Clikeman, T. T.; Dubceac, C.; Popov, A. A.; Chen, Y.-S.; Petrukhina, M. A.; Strauss, S. H.; Boltalina, O. V. Corannulene Molecular Rotor with Flexible Perfluorobenzyl Blades: Synthesis, Structure and Properties. Chem. Eur. J. 2015, 21, 94889492. 
(20) Chen, R.; Lu, R.-Q.; Shi, K.; Wu, F.; Fang, H.-X.; Niu, Z.-X.; Yan, X.-Y.; Luo, M.; Wang, X.-C.; Yang, C.-Y. et al. Corannulene Derivatives with low LUMO Levels and Dense Convex-Concave Packing for n-Channel Organic Field-Effect Transistors. Chem. Commun. 2015, 51, 13768-13771.

(21) Sanyal, S.; Manna, A. K.; Pati, S. K. Functional Corannulene: Diverse Structures, Enhanced Charge Transport, and Tunable Optoelectronic Properties. ChemPhysChem 2014, 15, 885-893.

(22) Sygula, A.; Saebø, S. $\pi-\pi$ Stacking of Curved Carbon Networks: The Corannulene Dimer. Int. J. Quantum Chem. 2009, 109, 65-72.

(23) Narita, K.; Okada, S. Geometric and Electronic Structures of Corannulene Polymers: Ultra Narrow Graphene Ribbons with Corrugation and Topological Defects. Chem. Phys. Lett. 2016, 650, 76-81.

(24) Obolensky, O. I.; Semenikhina, V. V.; Solov'yov, A. V.; Greiner, W. Interplay of Electrostatic and van der Waals Forces in Coronene Dimer. Int. J. Quantum Chem. 2007, 107, 1335-1343.

(25) Shi, X.-Q.; Van Hove, M. A.; Zhang, R.-Q. Survey of Structural and Electronic Properties of $\mathrm{C}_{60}$ on Close-Packed Metal Surfaces. J. Mater. Sci. 2012, 47, 7341-7355.

(26) Fasel, R.; Aebi, P.; Agostino, R. G.; Naumovic, D.; Osterwalder, J.; Santaniello, A.; Schlapbach, L. Orientation of Adsorbed C60 Molecules Determined via X-Ray Photoelectron Diffraction. Phys. Rev. Lett. 1996, 76, 4733-4736.

(27) Bauert, T.; Merz, L.; Bandera, D.; Parschau, M.; Siegel, J. S.; Ernst, K.-H. Building 2D Crystals from 5-Fold-Symmetric Molecules. J. Am. Chem. Soc. 2009, 131, 3460-3461.

(28) Zoppi, L.; Martin-Samos, L.; Baldridge, K. K. Effect of Molecular Packing on 
Corannulene-Based Materials Electroluminescence. J. Am. Chem. Soc. 2011, 133, $14002-14009$.

(29) Valenti, G.; Bruno, C.; Rapino, S.; Fiorani, A.; Jackson, E. A.; Scott, L. T.; Paolucci, F.; Marcaccio, M. Intense and Tunable Electrochemiluminescence of Corannulene. J. Phys. Chem. C 2010, 114, 19467-19472.

(30) Mack, J.; Vogel, P.; Jones, D.; Kaval, N.; Sutton, A. The Development of CorannuleneBased Blue Emitters. Org. Biomol. Chem. 2007, 5, 2448-2452.

(31) Kobryn, L.; Henry, W. P.; Fronczek, F. R.; Sygula, R.; Sygula, A. Molecular Clips and Tweezers with Corannulene Pincers. Tetrahedron Lett. 2009, 50, 7124-7127.

(32) Sandi, G.; Gerald, R. E.; Scanlon, L. G.; Johnson, C. S.; Klingler, R. J.; Rathke, J. W. Molecular Orbital and Li-7 NMR Investigation of the Influence of Curved Lattices in Lithium Intercalated Carbon Anodes. J. New Mat. Electrochem. Syst. 2000, 3, 13-19.

(33) Yokoi, H.; Hiraoka, Y.; Hiroto, S.; Sakamaki, D.; Seki, S.; Shinokubo, H. NitrogenEmbedded Buckybowl and its Assembly with $\mathrm{C}_{60}$. Nat. Commun. 2015, 6, 8215 .

(34) Zabula, A. V.; Filatov, A. S.; Spisak, S. N.; Rogachev, A. Y.; Petrukhina, M. A. A Main Group Metal Sandwich: Five Lithium Cations Jammed Between Two Corannulene Tetraanion Decks. Science 2011, 333, 1008-1011.

(35) Xie, Q.; Perez-Cordero, E.; Echegoyen, L. Electrochemical Detection of $\mathrm{C}_{60}^{6-}$ and $\mathrm{C}_{70}^{6-}$ : Enhanced Stability of Fullerides in Solution. J. Am. Chem. Soc. 1992, 114, 3978-3980.

(36) Bavdek, G.; Cossaro, A.; Cvetko, D.; Africh, C.; Blasetti, C.; Esch, F.; Morgante, A.; Floreano, L. Pentacene Nanorails on Au(110). Langmuir 2008, 24, 767-772.

(37) Fratesi, G.; Lanzilotto, V.; Stranges, S.; Alagia, M.; Brivio, G. P.; Floreano, L. High Resolution NEXAFS of Perylene and PTCDI: a Surface Science Approach to Molecular Orbital Analysis. Phys. Chem. Chem. Phys. 2014, 16, 14834-14844. 
(38) Giannozzi, P.; Baroni, S.; Bonini, N.; Calandra, M.; Car, R.; Cavazzoni, C.; Ceresoli, D.; Chiarotti, G. L.; Cococcioni, M.; Dabo, I. et al. QUANTUM ESPRESSO: a Modular and Open-Source Software Project for Quantum Simulations of Materials. J. Phys. Condens. Matter 2009, 21, 395502.

(39) Giannozzi, P.; Andreussi, O.; Brumme, T.; Bunau, O.; Nardelli, M. B.; Calandra, M.; Car, R.; Cavazzoni, C.; Ceresoli, D.; Cococcioni, M. et al. Advanced Capabilities for Materials Modelling with Quantum ESPRESSO. J. Phys. Condens. Matter 2017, 29, 465901.

(40) Klimes̆, J.; Bowler, D. R.; Michaelides, A. Chemical Accuracy for the van der Waals Density Functional. J. Phys. Condens. Matter 2010, 22, 022201.

(41) Monkhorst, H. J.; Pack, J. D. Special Points for Brillouin-Zone Integrations. Phys. Rev. $B$ 1976, 13, 5188 .

(42) Pehlke, E.; Scheffler, M. Evidence for Site-Sensitive Screening of Core Holes at the Si and Ge (001) Surface. Phys. Rev. Lett. 1993, 71, 2338-2341.

(43) Triguero, L.; Pettersson, L. G. M.; Agren, H. Calculations of Near-Edge X-RayAbsorption Spectra of Gas-Phase and Chemisorbed Molecules by Means of DensityFunctional and Transition-Potential Theory. Phys. Rev. B 1998, 58, 8097.

(44) Leetmaa, M.; Ljungberg, M.; Lyubartsev, A.; Nilsson, A.; Pettersson, L. Theoretical Approximations to X-Ray Absorption Spectroscopy of Liquid Water and Ice. J. Electron Spectrosc. Relat. Phenom. 2010, 177, 135-157.

(45) Gougoussis, C.; Calandra, M.; Seitsonen, A.; Mauri, F. First-Principles Calculations of X-Ray Absorption in a Scheme Based on Ultrasoft Pseudopotentials: From $\alpha$-Quartz to High-T ${ }_{c}$ Compounds. Phys. Rev. B 2009, 80, 075102. 
(46) Fratesi, G.; Lanzilotto, V.; Floreano, L.; Brivio, G. P. Azimuthal Dichroism in NearEdge X-ray Absorption Fine Structure Spectra of Planar Molecules. J. Phys. Chem. C 2013, 117, 6632-6638.

(47) Baby, A.; Fratesi, G.; Vaidya, S. R.; Patera, L. L.; Africh, C.; Floreano, L.; Brivio, G. P. Anchoring and Bending of Pentacene on Aluminum (001). J. Phys. Chem. C 2015, 119, $3624-3633$.

(48) Baby, A.; Lin, H.; Brivio, G. P.; Floreano, L.; Fratesi, G. Core-Level Spectra and Molecular Deformation in Adsorption: V-Shaped Pentacene on $\mathrm{Al}(001)$. Beilstein J. Nanotechnol. 2015, 6, 2242-2251.

(49) Ugolotti, A.; Harivyasi, S. S.; Baby, A.; Dominguez, M.; Pinardi, A. L.; López, M. F.; Martín-Gago, J. Á.; Fratesi, G.; Floreano, L.; Brivio, G. P. Chemisorption of Pentacene on Pt(111) with a Little Molecular Distortion. J. Phys. Chem. C 2017, 121, 2279722805 .

(50) Tersoff, J.; Hamann, D. Theory and Application for the Scanning Tunneling Microscope. Phys. Rev. Lett. 1983, 50, 1998.

(51) Cvetko, D.; Renzi, V. D.; Floreano, L.; Morgante, A.; Peloi, M.; Tommasini, F.; Cháb, V.; Prince, K. C. Vacancy Island Nucleation and Inverse Growth of InSb(110). Phys. Rev. B 1995, 51, 17957-17964.

(52) Michely, T.; Besocke, K.; Comsa, G. Observation of Sputtering Damage on Au(111). Surf. Sci. Lett. 1990, 230, L135-L139.

(53) Rose, M. K.; Mitsui, T.; Dunphy, J.; Borg, A.; Ogletree, D. F.; Salmeron, M.; Sautet, P. Ordered Structures of CO on Pd(1 11 1) Studied by STM. Surf. Sci. 2002, 512, 48-60.

(54) Lackinger, M.; Griessl, S.; Heckl, W. M.; Hietschold, M. Coronene on Ag(111) Investigated by LEED and STM in UHV. J. Phys. Chem. B 2002, 106, 4482-4485. 
(55) Floreano, L.; Cossaro, A.; Gotter, R.; Verdini, A.; Bavdek, G.; Evangelista, F.; Ruocco, A.; Morgante, A.; Cvetko, D. Periodic Arrays of Cu-Phthalocyanine Chains on $\mathrm{Au}(110)$. J. Phys. Chem. C 2008, 112, 10794-10802.

(56) Betti, M. G.; Gargiani, P.; Mariani, C.; Biagi, R.; Fujii, J.; Rossi, G.; Resta, A.; Fabris, S.; Fortuna, S.; Torelles, X. et al. Structural phases of ordered FePc-nanochains self-assembled on Au(110). Langmuir 2012, 28, 13232-13240.

(57) Li, H. I.; Pussi, K.; Hanna, K. J.; Wang, L. L.; Johnson, D. D.; Cheng, H.-P.; Shin, H.; Curtarolo, S.; Moritz, W.; Smardon, J. A. et al. Surface Geometry of $\mathrm{C}_{60}$ on $\mathrm{Ag}(111)$. Phys. Rev. Lett. 2009, 103, 056101.

(58) Chen, G.; Cooks, R. G.; Corpuz, E.; Scott, L. T. Estimation of the Electron Affinities of $\mathrm{C}_{60}$, Corannulene, and Coronene by Using the Kinetic Method. J. Am. Soc. Mass Spectrom. 1996, 7, 619-627.

(59) Löwdin, P.-O. On the Non-Orthogonality Problem Connected with the Use of Atomic Wave Functions in the Theory of Molecules and Crystals. J. Chem. Phys. 1950, 18, $365-375$.

(60) Zoppi, L.; Garcia, A.; Baldridge, K. K. Theoretical Investigation of the Binding Process of Corannulene on a Cu (111) Surface. J. Phys. Chem. A 2010, 114, 8864-8872.

(61) Baby, A.; Gruenewald, M.; Zwick, C.; Otto, F.; Forker, R.; van Straaten, G.; Franke, M.; Stadtmüller, B.; Kumpf, C.; Brivio, G. P. et al. Fully Atomistic Understanding of the Electronic and Optical Properties of a Prototypical Doped Charge-Transfer Interface. ACS Nano 2017, 11, 10495-10508.

(62) Perdew, J. P.; Burke, K.; Ernzerhof, M. Generalized Gradient Approximation Made Simple. Phys. Rev. Lett. 1996, 77, 3865. 
(63) Lovat, G.; Forrer, D.; Abadia, M.; Dominguez, M.; Casarin, M.; Rogero, C.; Vittadini, A.; Floreano, L. On-Surface Synthesis of a Pure and Long-Range-Ordered Titanium(IV)-Porphyrin Contact Layer on Titanium Dioxide. J. Phys. Chem. C 2017, 121, 13738-13746.

(64) Stöckl, Q. S.; Wu, T.-C.; Mairena, A.; Wu, Y.-T.; Ernst, K.-H. Erecting Buckybowls onto their Edge: 2D Self-Assembly of Terphenylcorannulene on the $\mathrm{Cu}(111)$ Surface. Faraday Discuss. 2017, 204, 429-437.

(65) Smerieri, M.; Pís, I.; Ferrighi, L.; Nappini, S.; Lusuan, A.; Vattuone, L.; Vaghi, L.; Papagni, A.; Magnano, E.; Valentin, C. D. et al. Private communication.

(66) Rocco, M. L. M.; Haeming, M.; Batchelor, D. R.; Fink, R.; Schöll, A.; Umbach, E. Electronic Relaxation Effects in Condensed Polyacenes: A High-Resolution Photoemission Study. J. Chem. Phys. 2008, 129, 074702.

(67) Li, W.-q.; Chang, Y.; Tian, W. Q.; Sun, X.-D. Temperature Dependence of Nonlinear Optical Properties in Li Doped Nano- Carbon Bowl Material. Appl. Phys. Lett. 2013, 102, 153307.

(68) Gao, B.; Liu, L.; Wang, C.; Wu, Z.; Luo, Y. Spectral Identification of Fullerene $\mathrm{C}_{82}$ Isomers. J. Chem. Phys. 2007, 12\%, 164314.

(69) Bassan, A.; Nyberg, M.; Luo, Y. Identifying Isomers of $\mathrm{C}_{78}$ by Means of X-Ray Spectroscopy. Phys. Rev. B 2002, 65, 165402.

(70) Cavalleri, M.; Odelius, M.; Nordlund, D.; Nilsson, A.; Pettersson, L. G. M. Half or Full Core Hole in Density Functional Theory X-Ray Absorption Spectrum Calculations of Water? Phys. Chem. Chem. Phys. 2005, 7, 2854.

(71) Nyberg, M.; Luo, Y.; Triguero, L.; Pettersson, L. G. M.; Ågren, H. Core-Hole Effects in X-Ray-Absorption Spectra of Fullerenes. Phys. Rev. B 1999, 60, 7956-7960. 
(72) Stöhr, J. NEXAFS Spectroscopy; Springer-Verlag Berlin Heidelberg, 1992; Vol. 25.

(73) O’Donnell, K. M.; Hedgeland, H.; Moore, G.; Suleman, A.; Siegl, M.; Thomsen, L.; Warschkow, O.; Schofield, S. R. Orientation and Stability of a bi-Functional Aromatic Organic Molecular Adsorbate on Silicon. Phys. Chem. Chem. Phys. 2016, 18, 2729027299 .

(74) Matsuura, A.; Thrupp, N.; Gonze, X.; Pouillon, Y.; Bruant, G.; Onida, G. The ETSF: An e-Infrastructure That Bridges Simulations and Experiments. Comput. Sci. Eng. 2012, 14, 22-32. 


\section{TOC Graphic}

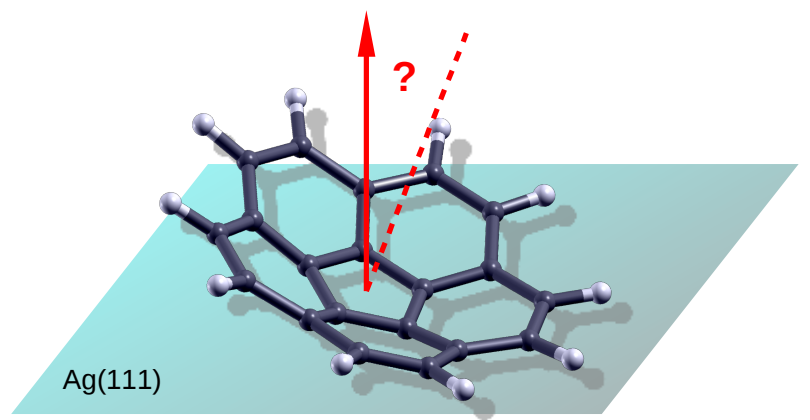

